\title{
Análise comparativa da alimentação de peixes (Teleostei) entre ambientes de marisma e de manguezal num estuário do sul do Brasil (Baía de Guaratuba, Paraná) ${ }^{1}$
}

\author{
Paulo de T. Chaves $^{2} \&$ Ana Lúcia Vendel ${ }^{3}$ \\ ${ }^{1}$ Contribuição número 1731 do Departamento de Zoologia, Universidade Federal do Paraná. \\ 2 Departamento de Zoologia, Universidade Federal do Paraná. Caixa Postal 19020, 81531-980 Curitiba, Paraná, Brasil. \\ E-mail:ptchaves@ufpr.br \\ ${ }^{3}$ Centro de Ciências Biológicas e Sociais Aplicadas, Universidade Estadual da Paraíba. Campus V, 58058-420 João Pessoa, \\ Paraíba, Brasil. E-mail: analuciavendel@uepb.edu.br
}

\begin{abstract}
Study on feeding habits in estuarine fish (Teleostei) comparatively between salt marshes and mangroves in southern Brazil (Guaratuba Bay). Diet of fish inhabiting shallow waters close to salt marshes and mangroves was analyzed in order to evaluate how different the influence of these environments on fish feeding habits is. The six studied species, the most abundant in these areas, are mainly planctyvores, however they showed particularities in each area. In salt marshes Atherinella brasiliensis (Quoy \& Gaimard, 1825) presented the largest number of food items and the lowest value of similarity regarding other species. In mangroves these attributes were presented by Anchoa januaria (Steindachner, 1879), the main species to feed on Brachyura and nonBrachyura Decapoda, and the only one in this area that includes Gammaridae in its diet. Anchoviella lepidentostole (Fowler, 1911) showed a large affinity with Anchoa lyolepis (Evermann \& Marsh, 1900) in salt marshes but a large one with Opisthonema oglinum (Le Sueur, 1818) and Harengula clupeola (Cuvier, 1829) in mangroves. Similar behaviour between salt marsh and mangrove has linked $H$. clupeola to $O$. oglinum, in which diet was composed almost integrally by Diatomacea and Copepoda. The ability of fish to change their feeding habits according to the environment is well-known worldwide; here it probably results from the availability of preys, which is supposed to be different between salt marshes and mangroves. However, it was also observed that, although food items change from salt marsh to mangrove, the relationships between species remain different in both areas, helping them to be abundant in the shallow estuarine waters.
\end{abstract}

KEY WORDS. Anchoa lyolepis; Anchoviella lepidentostole; Harengula clupeola; Opisthonema oglinum; Atherinella brasiliensis.

RESUMO. Estudou-se a composição da dieta de peixes em dois tipos de ambiente de áreas rasas estuarinas marisma e manguezal, objetivando avaliar se essas formações vegetais desencadeiam na ictiofauna respostas diferentes quanto à alimentação. As seis espécies avaliadas, as mais abundantes nessas áreas, mostraram-se predominantemente planctívoras, porém com particularidades quanto ao tipo de vegetação ocupada. Na marisma, Atherinella brasiliensis (Quoy \& Gaimard, 1825) apresentou a dieta com maior número de itens e menor similaridade em relação às demais espécies. No manguezal tal isolamento coube a Anchoa januaria (Steindachner, 1879), espécie com maior participação de Decapoda Brachyura e Decapoda não-Brachyura, e única que nesse ambiente incluiu Gammaridae na dieta. Anchoviella lepidentostole (Fowler, 1911) identificou-se com Anchoa lyolepis (Evermann \& Marsh, 1900) na marisma e com Opisthonema oglinum (Le Sueur, 1818) e Harengula clupeola (Cuvier, 1829) no manguezal. Uma situação comum a marisma e manguezal registrou-se entre $O$. oglinum e $H$. clupeola, espécies com dietas praticamente restritas a Diatomacea e Copepoda. Evidenciou-se o quanto as espécies são capazes de variar sua dieta com o ambiente, provavelmente em resposta à disponibilidade local. Mais que isso, porém, constatou-se que, seja na marisma, seja no manguezal, mesmo havendo mudança nos hábitos tróficos das espécies, cada uma delas mantém um padrão de diferenças em relação às demais que compõem a assembléia, fato que possivelmente assegura a abundância e coexistência entre elas nas áreas estuarinas rasas.

PALAVRAS-CHAVE. Anchoa lyolepis; Anchoviella lepidentostole; Harengula clupeola; Opisthonema oglinum; Atherinella brasiliensis.

Uma das evidências da relação existente entre os peixes e o ambiente onde se encontram manifesta-se nos hábitos tróficos. A dieta dos indivíduos depende de variáveis intrínse- cas a cada espécie, como anatomia bucal, exigências nutricionais e capacidade de detecção e apreensão, mas pode variar também, numa mesma etapa da vida, segundo a disponibilidade 
local de alimento (Kennish 1990). No ecossistema litorâneo uma demonstração desse fato foi relatada por CHAVEs \& UMBRIA (2003), envolvendo indivíduos de cinco espécies de Carangidae e Sciaenidae, de semelhante faixa de tamanho, coletados no interior do estuário e na plataforma adjacente. Entre os dois ambientes diferenças foram registradas em 50 a 90\% dos itens; numa mesma espécie dessas famílias a dieta pode incluir, por exemplo, insetos no interior do estuário e anfioxos e ofiuróides na plataforma. Outras diferenciações de dieta por ambiente foram relatadas por Cipólli (1990) numa espécie de manjuba do litoral sul de São Paulo, e por SHinnaKa et al. (2007) em assembléia de peixes de manguezal da Tailândia.

Estudos de alimentação de peixes estuarinos versam usualmente sobre descrições auto-ecológicas, ontogenéticas e de teias tróficas (BLABER 2000). O presente trabalho analisa comparativamente a composição da dieta de peixes entre dois microhabitats de um estuário. Analisam-se seis espécies de teleósteos comuns nas áreas rasas da Baía de Guaratuba, litoral do Estado do Paraná, um sistema margeado por formações de marisma e de manguezal. A marisma é vegetada pela gramínea Spartina alterniflora, compondo feições pioneiras que colonizam baixios areno-lodosos. Na região sua biomassa aérea alcança nível máximo no verão e mínimo no inverno (NetTo \& Lana 1999). O manguezal, composto por árvores de Rhizophora mangle, Laguncularia racemosa e Avicennia schaueriana, tem biomassa mais uniforme ao longo do ano. Marisma (Bonnet et al. 1994) e manguezal (VANNUCCI 1999) desempenham reconhecido papel na dinâmica de produção dos estuários. Sabe-se que em estuários a dieta dos peixes apresenta elevada plasticidade (EDGAR \& SHAW 1995), mas espécies com certo grau de especialização são noticiadas (Blaber 2000). Dessa forma, diferenças na dieta entre indivíduos quando em marisma e quando em manguezal, se demonstradas, ilustrarão não apenas a adaptabilidade das espécies a condições de micro-hábitats de um estuário, mas também o papel distinto que essas duas formações podem ter sobre a alimentação de peixes.

\section{MATERIAL E MÉTODOS}

As coletas foram realizadas bimestralmente entre abril de 1999 e fevereiro de 2000, no período diurno e de quadratura da baixa-mar. Fixaram-se dois locais da Baía de Guaratuba com profundidade inferior a dois metros, situando-se um deles local 1 - na cabeceira da Baía e o outro - local 2 - próximo à conexão entre a Baía e o mar, a $7 \mathrm{Km}$ do primeiro (Fig. 1). Em cada local três lances de arrasto de praia foram praticados em área adjacente à marisma, e outros três em área adjacente ao manguezal. A rede, abertura de malha $5 \mathrm{~mm}$, possuía $30 \mathrm{~m}$ de comprimento e $2,5 \mathrm{~m}$ de altura, e era tracionada manualmente mediante cerco em semicírculo contra a vegetação marginal. Imediatamente antes de cada arrasto foram mensurados na água de superfície a temperatura, a salinidade e o $\mathrm{pH}$.

Os peixes foram conservados em gelo até o laboratório, onde foram identificados, medidos (comprimento total) e então abertos para retirada do estômago, o qual foi fixado em formol 10\%. Descontados os estômagos vazios, analisou-se o conteúdo estomacal de 546 indivíduos (Tabs I e II) das seis espécies com maior abundância numérica: as manjubas Anchoa lyolepis (Evermann \& Marsh, 1900), A. januaria (Steindachner, 1879) e Anchoviella lepidentostole (Fowler, 1911) (Engraulidae); as sardinhas cascuda Harengula clupeola (Cuvier, 1829) e bandeira Opisthonema oglinum (Le Sueur, 1818) (Clupeidae); e o peixe-rei Atherinella brasiliensis (Quoy \& Gaimard, 1825) (Atherinopsidae).

$\mathrm{O}$ tratamento dos dados deu-se pelo método Freqüência de Ocorrência - porcentagem de estômagos contendo o item $i$ dentre os estômagos não-vazios analisados, e os resultados foram processados mediante classificação hierárquica não-ponderada (UPGMA) e o Índice de Similaridade Geral de Gower (Legendre \& Legendre 1984). Dessa análise excluiu-se o item sedimento, visto não ter sido considerado alimento. Para comparação entre as freqüências de ocorrência de cada item alimentar segundo o ambiente a a espécie, utilizou-se o teste de qui-quadrado: $\chi^{2}=2$ (FO-FE) $2 / \mathrm{FE}$, onde FO é a freqüência observada do item em questão na marisma ou no manguezal, e FE a freqüência esperada do mesmo, calculada pela média aritmética simples entre a freqüência observada na marisma e a freqüência observada no manguezal.

Exemplares-testemunho encontram-se depositados no Museu de História Natural Capão da Imbuia, em Curitiba, Paraná, sob tombos MHNCI 8998 a 9000.

\section{RESULTADOS}

Os valores médios de temperatura da água variaram entre $18,0^{\circ} \mathrm{C}$ (locais 1 e 2) em junho e $26,8^{\circ} \mathrm{C}$ (local 1 ) ou $28,8^{\circ} \mathrm{C}$ (local 2) em dezembro. Os médios de salinidade, entre 0,7 (abril) e 11,3 (junho) no local 1, e entre 18,0 (outubro) e 24,0 (junho) no local 2. E os médios de $\mathrm{pH}$, entre 7,25 (dezembro) e 8,63 (outubro) no local 1, e entre 8,07 (fevereiro) e 8,83 (junho) no local 2 (Tab. III). A fauna acompanhante dos arrastos compôsse, além de peixes, principalmente do Gastropoda Heleobia sp e de crustáceos Decapoda como Penaeidae - Farfantepenaeus sp. e Litopenaeus schmitti (Burkenroad, 1936), Palaemonidae Macrobrachium acanthurus (Wiegmann, 1836), e Portunidae Callinectes danae Smith, 1869 e C. sapidus Rathbun, 1896.

Plâncton predominou no conteúdo estomacal das seis espécies de peixes estudadas, com destaque em todas elas aos itens Copepoda e, secundariamente, Diatomacea (Tab. IV). Entre as diatomáceas predominou o grupo Bacillariophyta. Entre os insetos registraram-se fragmentos de Coleoptera, de Phloeothripidae (Tubulifera, Ordem Thysanoptera) e de Formicidae (Vespoidea) e Chalcidoidea, as duas últimas da Ordem Hymenoptera.

Todas as espécies apresentaram em sua dieta similaridade parcial de itens consumidos na marisma e no manguezal. Foram registrados nas duas formações: (I) em todas as espécies, Copepoda e Diatomacea; (II) em A. lyolepis, A. januaria e A. brasiliensis, Gastropoda e Bivalvia; (III) em A. januaria, A. lepidentostole e A. brasiliensis, Decapoda não-Brachyura; (IV) em

Revista Brasileira de Zoologia 25 (1): 10-15, March, 2008 


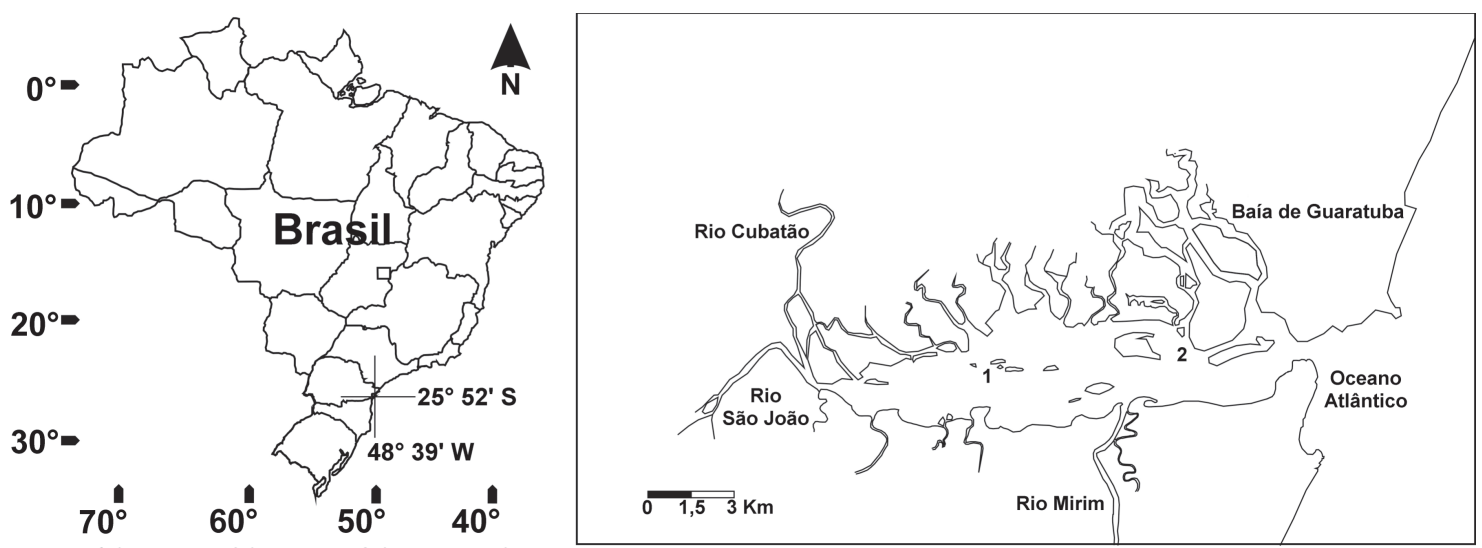

Figura 1. Localização da Baía de Guaratuba no litoral brasileiro e indicação dos locais 1 e 2 onde foram realizadas as amostragens.

Tabela I. Número de indivíduos analisados por espécie segundo o local e o ambiente, entre abril de 1999 e fevereiro de 2000 , no interior da Baía de Guaratuba.

\begin{tabular}{|c|c|c|c|c|}
\hline \multirow{2}{*}{ Espécies } & \multicolumn{2}{|c|}{ Local 1} & \multicolumn{2}{|c|}{ Local 2} \\
\hline & Manguezal & Marisma & Manguezal & Marisma \\
\hline Anchoa lyolepis (Evermann \& Marsh 1900) & - & - & 22 & 32 \\
\hline Anchoa januaria (Steindachner 1879) & 68 & 60 & 15 & 22 \\
\hline Anchoviella lepidentostole (Fowler 1911) & - & - & 5 & 16 \\
\hline Harengula clupeola (Cuvier 1829) & - & - & 18 & 37 \\
\hline Opisthonema oglinum (Le Sueur 1818) & - & 5 & 8 & 34 \\
\hline Atherinella brasiliensis (Quoy \& Gaimard 1825) & 12 & 48 & 84 & 60 \\
\hline
\end{tabular}

Tabela II. Número de indivíduos analisados por espécie segundo o mês, entre abril de 1999 e fevereiro de 2000, marisma e manguezal reunidos.

\begin{tabular}{lccccccc}
\hline \multirow{2}{*}{ Espécies } & \multicolumn{7}{c}{ Meses } \\
\cline { 2 - 8 } & Abr & Jun & Ago & Out & Dez & Fev \\
\hline Anchoa lyolepis & - & - & - & - & - & 54 \\
Anchoa januaria & 55 & 11 & 20 & 21 & 17 & 41 \\
Anchoviella lepidentostole & - & - & - & - & - & 21 \\
Harengula clupeola & - & - & - & 8 & 32 & 15 \\
Opisthonema oglinum & - & 12 & - & - & - & 35 \\
Atherinella brasiliensis & 13 & 58 & 15 & 14 & 54 & 50 \\
\hline
\end{tabular}

A. januaria, Brachiopoda e Decapoda Brachyura; (V) em A. lyolepis, Ostracoda; e (VI) em A. brasiliensis, Insecta. Nenhum item foi registrado apenas no manguezal. Isopoda e peixes ocorreram exclusivamente na marisma, mas em freqüência inferior a 3\% dos estômagos analisados de $A$. brasiliensis e $H$. clupeola (respectivamente). Sedimento esteve presente em todas as espécies na marisma e no manguezal (neste, exceto em $H$. clupeola). Sua freqüência foi maior em $A$. lepidentostole, espécie que dentre todas as espécies teve a menor ocorrência de Copepoda.
Entre marisma e manguezal houve semelhança na composição qualitativa da dieta, porém a freqüência de ingestão de cada item foi diferente entre ambos. De acordo com a similaridade (Fig. 2), na marisma $A$. brasiliensis isolou-se das demais espécies, apresentando a dieta com maior número de itens e o menor valor de similaridade $(0,42)$. Ela foi a espécie com a segunda menor participação relativa de Copepoda na marisma e a única a apresentar Polychaeta e Isopoda (Tab. IV). No manguezal tal isolamento coube a A. januaria (similaridade de 0,52), espécie cuja dieta mostrou combinação de número grande de itens, baixa representatividade de Decapoda não-Brachyura, proporção elevada de Copepoda, e - nesse ambiente - única que incluiu Decapoda Brachyura (Tab. IV). Outra distinção entre marisma e manguezal manifestou-se pelos diferentes grupamentos de A. lepidentostole: com A. lyolepis na marisma e com O. oglinum e H. clupeola no manguezal (Fig. 2). Situação comum a marisma e manguezal registrou-se quanto ao grupamento formado por $H$. clupeola e O. oglinum, espécies que nos dois ambientes tiveram uma dieta praticamente restrita a Copepoda e Diatomacea (Fig. 2 e Tab. IV).

A diferença na freqüência de ingestão dos itens foi confirmada pelos valores do teste de qui-quadrado. Eles indicam que cinco dos 13 itens alimentares foram consumidos com freqüên- 


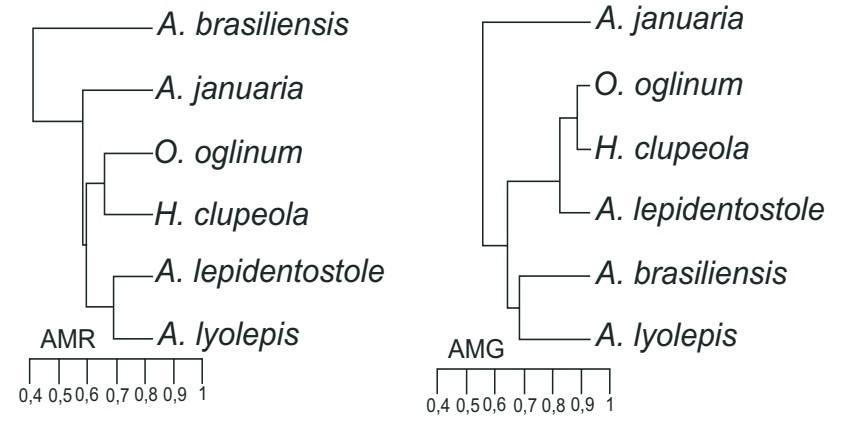

Figura 2. Grupamento dos itens alimentares, segundo o Coeficiente de Similaridade Geral de Gower (\%), em espécies amostradas em áreas associadas a marisma (AMR) e associadas ao manguezal (AMG) da Baía de Guaratuba. cias distintas entre manguezal e marisma. Com maior consumo no manguezal: Copepoda em H. clupeola, Polychaeta em $A$. brasiliensis, Diatomacea em O. oglinum, e Decapoda nãoBrachyura em A. lyolepis e A. lepidentostole. Com maior consumo na marisma: Copepoda em O. oglinum, Diatomacea em $A$. lepidentostole, Decapoda não-Brachyura em $H$. clupeola e Ostracoda em A. brasiliensis. Exceto por A. januaria, nas espécies estudadas ao menos um dos itens foi consumido com freqüência significativamente distinta entre os dois ambientes (Tab. IV).

\section{DISCUSSÃO}

As variáveis ambientais estudadas evidenciam que, em ambos os locais de amostragem, marisma e manguezal recebem influência direta de águas tanto continentais como marinhas. Valores de salinidade e $\mathrm{pH}$ mais baixos no local 1 que no

Tabela III. Valores de média mensal e desvio-padrão, entre parênteses, dos parâmetros abióticos mensurados entre abril de 1999 e fevereiro de 2000 nos dois locais estudados na Baía de Guaratuba, Paraná.

\begin{tabular}{|c|c|c|c|c|c|c|}
\hline \multirow{2}{*}{ Meses } & \multicolumn{3}{|c|}{ Local 1} & \multicolumn{3}{|c|}{ Local 2} \\
\hline & Temperatura & Salinidade & $\mathrm{pH}$ & Temperatura & Salinidade & $\mathrm{pH}$ \\
\hline Abril & $22,7(1,15)$ & $0,7(1,15)$ & $7,67(0,06)$ & $22,3(0,58)$ & $18,7(1,15)$ & $8,43(0,06)$ \\
\hline Junho & $18,0(0,00)$ & $11,3(1,15)$ & $8,43(0,06)$ & $18,0(0,00)$ & $24,0(0,00)$ & $8,83(0,29)$ \\
\hline Agosto & $19,3(0,58)$ & $10,0(0,00)$ & $8,27(0,12)$ & $20,3(0,58)$ & $19,3(2,31)$ & $8,63(0,06)$ \\
\hline Outubro & $20,3(0,58)$ & $3,3(1,15)$ & $8,63(0,67)$ & $21,3(0,58)$ & $18,0(8,72)$ & $8,13(0,12)$ \\
\hline Dezembro & $26,8(1,26)$ & $5,3(3,06)$ & $7,25(0,26)$ & $28,8(0,29)$ & $22,3(1,53)$ & $8,32(0,13)$ \\
\hline Fevereiro & $26,3(0,58)$ & $4,7(4,62)$ & $7,47(0,12)$ & $26,0(0,00)$ & $22,0(3,46)$ & $8,07(0,12)$ \\
\hline
\end{tabular}

Tabela IV. Freqüência de ocorrência dos itens registrados no conteúdo estomacal de peixes coletados entre abril de 1999 e fevereiro de 2000 nas áreas rasas da Baía de Guaratuba. Valores em negrito indicam diferença significativa $(\mathrm{g} . \mathrm{l} .=1 ; \alpha=0,05)$ na freqüência de itens alimentares entre manguezal (AMG) e marisma (AMR). (CT) Amplitude de tamanho (cm), (Dn) Decapoda não-Brachyura, (Db) Decapoda Brachyura. Entre parênteses se encontra o tamanho da amostra.

\begin{tabular}{|c|c|c|c|c|c|c|c|c|c|c|c|c|}
\hline \multirow{2}{*}{ Táxons/itens } & \multicolumn{2}{|c|}{$\begin{array}{c}\text { A. lyolepis } \\
\text { (CT: } 3,6-8,5)\end{array}$} & \multicolumn{2}{|c|}{$\begin{array}{c}\text { A. januaria } \\
\text { (CT: } 4,5-9,8)\end{array}$} & \multicolumn{2}{|c|}{$\begin{array}{l}\text { A. lepidentostole } \\
\text { (CT: } 4,0-7,9)\end{array}$} & \multicolumn{2}{|c|}{$\begin{array}{c}\text { H. clupeola } \\
\text { (CT: } 4,5-12,8)\end{array}$} & \multicolumn{2}{|c|}{$\begin{array}{c}\text { O. oglinum } \\
\text { (CT: } 6,0-11,0) \\
\end{array}$} & \multicolumn{2}{|c|}{$\begin{array}{l}\text { A. brasiliensis } \\
\text { (CT: } 5,9-15,1)\end{array}$} \\
\hline & $\begin{array}{l}\text { AMG } \\
(22)\end{array}$ & $\begin{array}{l}\text { AMR } \\
(32)\end{array}$ & $\begin{array}{l}\text { AMG } \\
(83)\end{array}$ & $\begin{array}{l}\text { AMR } \\
(82)\end{array}$ & $\begin{array}{l}\text { AMG } \\
(05)\end{array}$ & $\begin{array}{l}\text { AMR } \\
(16)\end{array}$ & $\begin{array}{l}\text { AMG } \\
(18)\end{array}$ & $\begin{array}{l}\text { AMR } \\
(37)\end{array}$ & $\begin{array}{l}\text { AMG } \\
(08)\end{array}$ & $\begin{array}{l}\text { AMR } \\
(39)\end{array}$ & $\begin{array}{l}\text { AMG } \\
(96)\end{array}$ & $\begin{array}{l}\text { AMR } \\
\text { (108) }\end{array}$ \\
\hline Copepoda & 40,9 & 56,3 & 60,2 & 52,4 & 20,0 & 31,3 & 77,8 & 54,1 & 50,0 & 74,4 & 44,8 & 50,9 \\
\hline Polychaeta & - & - & - & - & - & - & - & - & - & - & 11,5 & 0,9 \\
\hline Diatomacea & 22,7 & 18,8 & 9,6 & 11,0 & 20,0 & 50,0 & 22,2 & 32,4 & 50,0 & 23,1 & 12,5 & 15,7 \\
\hline Crustacea Dn & 4,5 & - & 4,8 & 11,0 & 60,0 & 18,8 & - & 10,8 & - & - & 2,1 & 4,6 \\
\hline Crustacea Db & - & - & 6,0 & 3,7 & - & - & - & - & - & - & - & 1,9 \\
\hline Insecta & - & - & - & - & - & - & - & - & - & - & 10,4 & 7,4 \\
\hline Gastropoda & 13,6 & 12,5 & 10,8 & 11,0 & - & - & - & - & - & - & 10,4 & 4,6 \\
\hline Bivalvia & 9,1 & 9,4 & 2,4 & 1,2 & - & - & - & - & - & - & 6,3 & 2,8 \\
\hline Ostracoda & 9,1 & 3,1 & - & 2,4 & - & - & - & - & - & - & - & 8,3 \\
\hline Brachiopoda & - & - & 6,0 & 3,7 & - & - & - & - & - & 2,6 & 2,1 & - \\
\hline Gammaridae & - & - & - & 3,7 & - & - & - & - & - & - & - & 0,9 \\
\hline Isopoda & - & - & - & - & - & - & - & - & - & - & - & 1,9 \\
\hline Peixe & - & - & - & - & - & - & - & 2,7 & - & - & - & - \\
\hline Sedimento* & 13,3 & 8,9 & 7,4 & 4,4 & 53,3 & 13,3 & - & 4,4 & 6,0 & 15,0 & 17,3 & 9,3 \\
\hline
\end{tabular}

\footnotetext{
* Não considerado item alimentar.
} 
local 2 traduzem a proximidade dos rios costeiros, com variações sazonais que refletem o ritmo de chuvas sobre a bacia versante. Assim, maior acidez da água foi registrada em dezembro e fevereiro, período quente e chuvoso com forte carreamento de matéria continental para o interior da Baía (Chaves \& Bouchereau 1999). Em contraste, a semelhança de temperatura da água entre ambos os locais revela que esta variável é menos suscetível que a salinidade e o pH às alterações decorrentes do regime pluviométrico.

Num estudo dessa natureza, com forte caráter comparativo, restrições de ordem técnica podem influenciar na interpretação dos resultados. O grau de detalhamento no reconhecimento dos itens - se no nível de ordem ou de família, por exemplo não parece ter sido um deles. Afinal, se mesmo nos níveis empregados - ordem, família - particularidades em marisma ou manguezal foram detectadas, mais ainda o teriam sido se a identificação tivesse ocorrido no nível de gênero ou de espécie. Quanto à representatividade espacial dos exemplares analisados, foi apenas no local 2 que a maioria das espécies ocorreu simultaneamente em ambos os micro-hábitats, mas assegurou-se a inclusão de indivíduos de mesma faixa de tamanho e das duas formações vegetais em épocas do ano semelhantes. Quanto à representatividade temporal, exemplares de $A$. lyolepis e $A$. lepidentostole foram coletados somente no verão; mesmo assim, a relação entre dietas, expressa pelo Índice de Similaridade, foi diferente entre marisma e manguezal tanto entre essas duas espécies, como entre elas e as demais aqui estudadas.

A natureza da dieta das seis espécies não diferiu em Guaratuba daquela já registrada para as mesmas noutras regiões. SERgipense et al. (1999) relatam em Anchoa januaria do litoral fluminense uma dieta baseada em Copepoda, especialmente Harpacticoida; Cipólur (1990) registra em Anchoviella lepidentostole da foz do Rio Ribeira do Iguape (São Paulo) Copepoda e Decapoda, porém maiores proporções de Insecta, Ostracoda e Cladocera; Ortaz et al. (1996), ao estudar a dieta de Harengula clupeola da costa da Venezuela, relatam sobretudo Copepoda e - secundariamente - larvas de outros Crustacea, porém incluindo peixes; CARR \& ADAMs (1973) registram na dieta de Opisthonema oglinum da Florida zooplâncton, notadamente Copepoda; e Hostim-SiLvA et al. (1995), na dieta de Atherinella brasiliensis da Lagoa da Conceição, Santa Catarina, principalmente microcrustáceos, Insecta e Diatomacea.

A presença recorrente de sedimento no conteúdo estomacal das espécies sinaliza atividade alimentar nas águas rasas e calmas que banham as formações vegetais estudadas. Entretanto, a ingestão de sedimento foi mais acentuada naquela espécie (A. lepidentostole) em que o consumo de Copepoda - o principal componente do zooplâncton - foi menos expressiva. Estima-se que o modo de obtenção de alimento por essa espécie de manjuba favorize itens que estão dispostos próximo ao sedimento, como os camarões.

A dominância de Copepoda entre os itens alimentares deve estar associada à sua disponibilidade no ambiente, visto que re- presenta mais de 90\% do zooplâncton na vizinha Baía de Paranaguá (LANa et al. 2001). Segundo RoberTson et al. (1988), em habitats de marisma e mangue, principalmente nas zonas estuarinas mais internas, o zooplâncton é abundante. Embora a maioria das espécies estudadas pertença à guilda das planctívoras, todas evidenciaram plasticidade para compor sua alimentação com diferenças no consumo de itens entre marisma e manguezal. Esse fato ilustra a adaptabilidade trófica da ictiofauna aos microhábitats de um ambiente estuarino, assim como a importância que essas vegetações têm para a vida dos peixes de estuários. SHINNAKA et al. (2007) relatam uma maior abundância de peixes zooplanctófagos em áreas adjacentes a manguezal suprimido que a manguezal íntegro, nos quais a bentivoria predomina. Nos peixes de águas rasas espera-se dominância de hábito planctófago, em contraste com o hábito bentófago classicamente conhecido na ictiofauna demersal (Longhurst 1957, Blaber 2000). De fato, conforme discutido por SASEKUMAR et al. (1992) е THOLLOT (1996), dentre outros, em geral assume-se que o ponto de partida para obtenção de energia pelos peixes dos estuários tipo manguezal é a detritivoria. Entretanto, conforme verificado no presente trabalho, em se tratando de peixes de águas rasas, não-demersais, o hábito zooplanctófago predomina tanto na marisma como no manguezal, o que sublinha o papel da produção primária como base da cadeia trófica para essa significativa parcela da ictiofauna estuarina.

\section{AGRADECIMENTOS}

Aos pescadores João C. da Costa e Itamar Costa, auxiliares no trabalho de campo. Também aos dois Consultores da Revista, por sua contribuição. Licenças de coleta: IBAMA 02017.002797/00-63 e Instituto Ambiental do Paraná 3.940.329-3. Financiamento: CNPq - bolsas Produtividade em Pesquisa (PTC) e Doutorado (ALV).

\section{LITERATURA CITADA}

Blaber, S.J.M. 2000. Tropical estuarine fishes. ecology, exploitation and conservation. Oxford, Blackwell Science, 372p.

BonNeT, B.R.P.; P.C. LANA \& C. GuIss. 1994. Influência da gramínea Spartina alterniflora sobre a distribuição e densidade de Neritina virginea (Gastropoda: Neritidae) em marismas da Baía de Paranaguá (Paraná, Brasil). Nerítica 8 (1-2): 99-108.

CARR, W.E.S. \& C.A. ADAMs. 1973. Food habitats of juvenile marine fishes occupying seagrass beds in the estuarine zone near Crystal River, Florida. Transactions of American Fisheries Society 102 (3): 511-539.

Chaves, P.T. \& J.-L. Bouchereau. 1999. Biodiversity and dynamics of ichthyic communities in the mangrove of Guaratuba, Brazil. Oceanologica Acta 22 (3): 353-364.

Chaves, P.T. \& S.C. Umbria. 2003. Changes in the diet composition of transitory fishes in coastal systems, estuary and continental shelf. Brazilian Archives of Biology and Technology 46 (1): 41-46. 
Cipólli, M.N. 1990. Regime alimentar, p. 17-18. In: A. Bendazoli \& C.L.D.B. Rossi-Wongtschowski (Eds). A manjuba no Rio Ribeira de Iguape: biologia, comportamento e avaliação do estoque. São Paulo, IBAMA/IOUSP/IP-AS/SEMA, 125p.

EDGAR, G.J. \& C. SHAW. 1995. The production and trophic ecology of shallow-water fish assemblages in southern Australia. III. General relationships between sediments, seagrass, invertebrates and fishes. Journal of Experimental Marine, Biology and Ecology 194: 53-81.

Hostim-Silva, M.; L. Clezar; G.C. Ribeiro \& C. Machado. 1995. Estrutura populacional de Xenomelaniris brasiliensis (Quoy \& Gaimard, 1824) (Osteichthyes-Atherinidae) na Lagoa da Conceição, SC, Brasil. Arquivos de Biologia e Tecnologia 38 (3): 949-960.

Kennish, M.J. 1990. Ecology of estuaries. Boston, CRC Press, 391p.

Lana, P.C.; E. Marone; R.M. Lopes \& E.C. Machado. 2001. The subtropical estuarine complex of Paranaguá Bay, Brazil, p. 132-145. In: U. Seeliger \& B. KJerfve (Eds). Coastal marine ecosystems of Latin American. Ecological Studies 144. Berlin, Springer Velrag, 350p.

Legendre, L. \& P. Legendre. 1984. Ecologie numérique: le traitement multiple des donnée écologiques. Paris, Masson PUQ, 270p.

Longhurst, A.R. 1957. The food of the demersal fish of a West African Estuary. Journal of Animal Ecology 26: 369-389.
NetTo, S.A. \& P.C. Lana. 1999. The role of above- and belowground components of Spartina alterniflora (Loisel) and detritus biomass in structuring macrobenthic associations of Paranaguá Bay (SE Brazil). Hydrobiologia 400: 167-177.

Ortaz, M.; M.E. Rocha \& J.M. Posada. 1996. Food habits of the sympatric fishes Harengula humeralis and H. clupeola (Clupeidae) in the Archipélago de Los Roques National Park, Venezuela. Caribbean Journal of Science 32 (1): 26-32.

Robertson, A.I.; P. Dixon \& P.A. Daniel. 1988. Zooplankton dynamics in mangrove and other nearshore habitats in tropical Australia. Marine Ecology Progress Series 43: 139-150.

Sasekumar, A.; V.C. Chong; M.U. Leh \& R. D’Cruz. 1992. Mangroves as a habitat for fish and prawns. Hydrobiologia 247: 195-207.

Sergipense, S.; E.P. Caramaschi \& I. Sazima, 1999. Morfologia e hábitos alimentares de duas espécies de Engraulidae (Teleostei, Clupeiformes) na Baía de Sepetiba, Rio de Janeiro. Revista Brasileira de Oceanografia 47 (2): 173-188.

Shinnaka, T.; M. Sano; K. Ikejima; P. Tongnunu; M. Horinouchi \& H. KurokURA. 2007. Effects of mangrove deforestation on fish assemblage at Pak Phanang Bay, Southern Thailand. Fisheries Science 73: 862-870.

ТноцLот, Р. 1996. Les poissons de mangrove du lagoon sudouest de Nouvelle-Calédonie. Paris, Orstom Éditions, 321p.

VANnUCCI, M. 1999. Os manguezais e nós. São Paulo, EDUSP, $233 p$.

Received in 13.IX.2007; accepted in 26.II.2008. 